Dhaka Univ. J. Sci. 65(1): 61-66, 2017 (January)

\title{
Numerical Solution of Nonlinear Fredholm Integral Equations by Using NKM and ADM
}

\author{
M. M. Hasan ${ }^{*}$ and M. A. Matin
}

\author{
Department of Mathematics, Dhaka University, Dhaka-1000, Bangladesh
}

(Received: 13 June 2016; Accepted: 1 November 2016)

\begin{abstract}
In this paper, we present a numerical method to solve a non-linear Fredholm integral equations. We intend to approximate the solution of this equation by Newton-Kantorovich-quadrature method and Adomian Decomposition method compare both the methods accurately for solving the non-linear Fredholm integral equation.
\end{abstract}

Keywords: Non-linear Fredholm integral equation, Newton-Kantorovich method, Adomian Decomposition Method, He's Polynomials, Approximate Solutions, and MATHEMATICA v9 software.

\section{Introduction}

No one disagrees on the importance of integral equations in our practical and scientific life. In fact, with these equations, we can identify various natural phenomena which may confront us. Integral equations are of high applicability in different areas of applied mathematics, physics, and engineering ${ }^{5}$. In particular, they are widely used as mathematical models of many problems such as in mechanics, geophysics, electricity and magnetism, hereditary phenomena in biology, quantum mechanics, and mathematical economics. The Fredholm integral equation is of widespread use in many realms of engineering and applied mathematics. Theory and application of integral equations are important subjects in the different branches of science and its advantage is witnessed by the increasing frequency in the literature and in many recent texts on methods of advanced applied mathematics.

Consider the general form non-linear Fredholm integral equation of the second kind

$$
y(x)=f(x)+\lambda \int_{a}^{b} K(x, t, y(t)) d t, a \leq x \leq b
$$

where $y(t)$ is the unknown solution, $a$ and $b$ is a real constants. The kernel $K(x, t, y(t))$ and $f(x)$ are known smooth functions on $R^{2}$ and $R$ respectively. The parameter $\lambda$ is real (or complex) known as the eigenvalue when $\lambda$ is a real parameter. In section $\mathbf{V}$, the application of the methods on numerical example shows its accuracy and efficiency.

\section{The Newton-Kantorovich Method}

An effective method that makes it possible to overcome the indicated complications is the Newton-Kantorovich method. The main objective of this method is the solution of nonlinear integral equations of the second kind with constant limits of integration. Nevertheless, this method is useful in the solution of many problems for the Volterra equations and makes it possible to significantly increase the rate of convergence compared with the successive approximation method.

The nonlinear integral equations with constant integration limits can be represented in the form

$$
y(x)=f(x)+\lambda \int_{a}^{b} K(x, t, y(t)) d t
$$

\footnotetext{
*Author for correspondence. e-mail: mohedul.math@gmail.com
}

$$
a \leq x \leq b
$$

This method is applied to find a solution for non-linear Fredholm integral equation of the second kind using an iteration process as follows ${ }^{7}$

$$
\begin{gathered}
y_{k}(x)=y_{k-1}(x)+\varphi_{k-1}(x) \\
\varphi_{k-1}(x)=\varepsilon_{k-1}(x) \\
+\int_{a}^{b} K_{y}\left(x, t, y_{k-1}(t)\right) \varphi_{k-1}(t) d t \\
\varepsilon_{k-1}(x)=f(x)-y_{k-1}(x) \\
+\int_{a}^{b} K\left(x, t, y_{k-1}(t)\right) d t
\end{gathered}
$$

where $K_{y}(x, t, y)=\frac{\partial}{\partial y} K(x, t, y)$.

From $(2 \mathrm{a})-(2 \mathrm{c})$, we have

$$
\begin{aligned}
& \varphi_{k-1}(x)=f(x)-y_{k-1}(x)+ \\
& +\int_{a}^{b} K\left(x, t, y_{k-1}(t)\right) d t \\
& +\int_{a}^{b} K_{y}\left(x, t, y_{k-1}(t)\right) \varphi_{k-1}(t) d t
\end{aligned}
$$

Now, we approximate the two integrals on the right side by one of the numerical integration formula such as repeated Simpson, repeated trapezoid or Gauss methods, so we get

$$
\begin{aligned}
\varphi_{k-1}(x) & =f(x)-y_{k-1}(x) \\
+ & \sum_{j=0}^{n} w_{j} K\left(x, x_{j}, y_{k-1}\left(x_{j}\right)\right) \\
+ & \sum_{j=0}^{n} w_{j} K_{y}\left(x, x_{j}, y_{k-1}\left(x_{j}\right)\right) \varphi_{k-1}\left(x_{j}\right)
\end{aligned}
$$

By substituting $x=x_{i}$ for $i=0,1,2, \ldots, n$, we obtain the following system:

$$
\begin{aligned}
\varphi_{k-1}\left(x_{i}\right) & =f\left(x_{i}\right)-y_{k-1}\left(x_{i}\right) \\
+ & \sum_{j=0}^{n} w_{j} K\left(x_{i}, x_{j}, y_{k-1}\left(x_{j}\right)\right)
\end{aligned}
$$




$$
\begin{gathered}
+\sum_{j=0}^{n} w_{j} K_{y}\left(x_{i}, x_{j}, y_{k-1}\left(x_{j}\right)\right) \varphi_{k-1}\left(x_{j}\right), \\
=0,1,2, \ldots \ldots n .
\end{gathered}
$$

Since $\varphi_{k-1}(x)=y_{k}(x)-y_{k-1}(x)$, we have

$$
\begin{aligned}
& y_{k}\left(x_{i}\right)-y_{k-1}\left(x_{i}\right)=f\left(x_{i}\right)-y_{k-1}\left(x_{i}\right) \\
& +\sum_{j=0}^{n} w_{j} K\left(x_{i}, x_{j}, y_{k-1}\left(x_{j}\right)\right) \\
& +\sum_{j=0}^{n} w_{j} K_{y}\left(x_{i}, x_{j}, y_{k-1}\left(x_{j}\right)\right)\left[y_{k}\left(x_{j}\right)\right. \\
& \left.\quad-y_{k-1}\left(x_{j}\right)\right], i=0,1,2, \ldots . . n .
\end{aligned}
$$

Therefore, we get

$$
\begin{aligned}
y_{k}\left(x_{i}\right) & =f\left(x_{i}\right)+\sum_{j=0}^{n} w_{j} K\left(x_{i}, x_{j}, y_{k-1}\left(x_{j}\right)\right) \\
+ & \sum_{j=0}^{n} w_{j} K_{y}\left(x_{i}, x_{j}, y_{k-1}\left(x_{j}\right)\right) \\
& \times\left[y_{k}\left(x_{j}\right)-y_{k-1}\left(x_{j}\right)\right], i=0,1,2, \ldots, n
\end{aligned}
$$

Now, we let

$$
\begin{gathered}
\left(F^{(k-1)}\right)_{i+1}=f\left(x_{i}\right) \\
+\sum_{j=0}^{n} w_{j}\left[K\left(x_{i}, x_{j}, y_{k-1}\left(x_{j}\right)\right)\right. \\
\left.-K_{y}\left(x_{i}, x_{j}, y_{k-1}\left(x_{j}\right)\right) y_{k-1}\left(x_{j}\right)\right] \\
, i=0,1,2, \ldots, n \\
\left(A^{(k-1)}\right)_{i+1, j+1}=w_{j} K_{y}\left(x_{i}, x_{j}, y_{k-1}\left(x_{j}\right)\right) \\
, i, j=0,1,2, \ldots, n \\
\left(Y^{(k)}\right)_{i+1}=y_{k}\left(x_{i}\right), \quad i,=0,1,2, \ldots, n
\end{gathered}
$$

To obtain the following sequence of linear systems. Thus, solving the following system we are able to solve this kind of equation:

$$
\left(I-A^{(k-1)}\right) Y^{(k)}=F^{(k-1)}, k=1,2,3, \ldots
$$

For this purpose, we first consider an initial solution $y_{0}$, so $\left(Y^{(0)}\right)_{i}=y_{0}\left(x_{i}\right)$ and we can construct $F^{(0)}$ and $A^{(0)}$, then by solving the system $\left(I-A^{(0)}\right) Y^{(1)}=F^{(0)}$, we obtain $Y^{(1)}$. By repeating this procedure we obtain the values $Y^{(0)}, Y^{(1)}, Y^{(2)}, \ldots \ldots \ldots . Y^{(m)}$ for selected $m \in N$.

\section{Introduction to Adomian Polynomial}

The Adomian decomposition method is a technique for solving functional equations in the form

$$
y(x)=f(x)+G(y)
$$

In some functional space, say $G$. The solution $y$ is considered as the summation of a series, say;

$$
y(x)=\sum_{i=0}^{\infty} y_{i}(x)
$$

The nonlinear term $G(y)$ as the summation of a series, say;

$$
G(y)=\sum_{k=0}^{\infty} A_{k}\left(y_{0}, y_{1}, y_{2}, \ldots, y_{k}\right)
$$

where $A_{k}$ 's, called Adomian polynomials has been introduced by Adomian himself by the formula

$$
A_{k}\left(y_{0}, y_{1}, \ldots ., y_{k}\right)=\frac{1}{k !} \frac{d^{k}}{d p^{k}}\left[G\left(\sum_{i=0}^{\infty} p^{i} y_{i}\right)\right]_{p=0},
$$$$
k=0,1,2, \ldots
$$

Other authors have suggested different Algorithm for computing Adomian polynomials ([2], [3]).

\section{The Adomian Decomposition Method}

In the recent earlier, a lot of researchers ([1], [2], and [9]) have expressed their interest in the study of Adomian decomposition method (ADM) for several scientific models. Adomian asserted that the ADM provides an efficient and computationally worthy method for generating approximate series solution for a large class approximate series solution for a large class of differential as well as integral equations. Consider the general form of non-linear Fredholm integral equation of the second kind

$$
\begin{gathered}
y(x)=f(x)+\lambda \int_{a}^{b} K(x, t) G(y(t)) d t, \\
a \leq x \leq b
\end{gathered}
$$

where $y(x)$ is the unknown solution, $a$ and $b$ are real constants. The kernel $K(x, t)$ and $f(x)$ are known smooth functions on $R^{2}$ and $R$ respectively. The parameter $\lambda$ is a real (or complex) number known as the eigenvalue when $\lambda$ is a real parameter, and $G$ is a non-linear function of $y$.

Assume that the solution of equation (4) can be written in the form

$$
\begin{gathered}
y=\sum_{i=0}^{\infty} p^{i} y_{i}(x) \\
=y_{0}+p^{1} y_{1}+p^{2} y_{2} \\
+p^{3} y_{3}+\cdots
\end{gathered}
$$

The comparisons of like powers of $p$ give solutions of various orders and the best approximation is

$$
y=\lim _{p \rightarrow 1} \sum_{i=0}^{\infty} p^{i} y_{i}(x)=y_{0}+y_{1}+y_{2}+. .
$$


The nonlinear term $G(y)$ can be expressed in He's polynomials ([3], [4]) as

$$
\begin{aligned}
G(y)= & \sum_{k=0}^{\infty} p^{k} H_{k}\left(y_{0}, y_{1}, y_{2}, \ldots, y_{k}\right) \\
= & H_{0}\left(y_{0}\right)+p^{1} H_{1}\left(y_{0}, y_{1}\right)+\cdots \\
& +p^{k} H_{k}\left(y_{0}, y_{1}, \ldots, y_{k}\right)
\end{aligned}
$$

where $H_{k}$ 's are the so called He's polynomials which can be calculated by using the formula

$$
\begin{aligned}
H_{k}\left(y_{0}, y_{1}, ., y_{k}\right) & =\frac{1}{k !} \frac{d^{k}}{d p^{k}}\left[G\left(\sum_{i=0}^{k} p^{i} y_{i}\right)\right], \\
k & =0,1,2 \ldots .
\end{aligned}
$$

Using (4b), (4c) and (4d) into (4a), we have

$$
\begin{aligned}
& \sum_{i=0}^{\infty} p^{i} y_{i}(x) \\
& =f(x)+\lambda \int_{a}^{b} K(x, t) \sum_{j=0}^{\infty}\left(p^{j} H_{j}\right) d t
\end{aligned}
$$

Equating the term with identical power of $p$ in equation (4e),

$$
\begin{aligned}
& p^{0}: y_{0}(x)=f(x) \\
& p^{1}: y_{1}(x)=\lambda \int_{a}^{b} K(x, t) H_{0}(t) d t \\
& p^{2}: y_{2}(x)=\lambda \int_{a}^{b} K(x, t) H_{1}(t) d t
\end{aligned}
$$

and in general form we have

$$
\left\{\begin{array}{c}
y_{0}(x)=f(x) \\
y_{k+1}(x)=\lambda \int_{a}^{b} K(x, t) H_{k}(t) d t \\
k=0,1,2, \ldots
\end{array}\right.
$$

Using the recursive scheme $(4 f)$, the $n$-term approximation series solution can be obtained as follows:

$$
\varphi_{n}(x)=\sum_{j=0}^{n} y_{j}(x)
$$

\section{Numerical Implementations}

In this section, we will apply the Newton-Kantorovich method and Adomian decomposition method to compute a numerical solution for a non-linear integral equation of the Fredholm type. Then we will compare between the results which we obtain by the numerical solution method and the results of the exact solution.

Numerical solutions of Fredholm integral equation by using the Newton-Kantorovich method

In this section, we will apply Newton-Kantorovich method to a nonlinear integral equation of the Fredholm type of the form (1) in order to find a numerical solution. To illustrate this, we consider the following example. For calculating the results in table, we use MATHEMATICA v9 software.

\section{Example}

Consider the following nonlinear Fredholm integral equation by applying

(a) The Newton-Kantorovich method and

(b) Adomian decomposition method

$$
\begin{gathered}
y(x)=\sin (\pi x) \\
+\frac{1}{5} \int_{0}^{1} \cos (\pi x) \sin (\pi t)(y(t))^{3} d t \\
\quad x \in[0,1]
\end{gathered}
$$

Solution

\section{(a) Newton-Kantorovich method}

The exact solution of the equation is

$y(x)=\sin (\pi x)+\frac{20-\sqrt{391}}{3} \cos (\pi x)([6],[7]$, and [8]).

Now first of all, for the initial approximation we take

$$
y_{0}(x)=\sin (\pi x)
$$

By repeating the previous processes, we stop the iteration at the third step. So we have

$$
\mathrm{y}(x)=\sin (\pi x)+\frac{123578799787}{163840000000} \cos (\pi x) .
$$

The table below shows the approximate solutions obtained by applying the Newton-Kantorovich method for three iterations according to the value of $x$, which is restrained between zero and one. We compared those results with the results which were obtained by the exact solution for this example, where the results are very close. Moreover, we can see the convergence of solutions in Figure 1 which is presented clearly. 
Table 1. Numerical and exact solutions of integral equation (5)

\begin{tabular}{|c|c|c|c|}
\hline Nodes & Exact values & Approximate values & Absolute Error \\
\hline 0.00 & 0.07542668890493687 & 0.07542663444213867 & $5.44628 \times 10^{-8}$ \\
\hline 0.05 & 0.23093252624133365 & 0.23093247244906287 & $5.37923 \times 10^{-8}$ \\
\hline 0.10 & 0.38075203836055493 & 0.38075198656335585 & $5.17972 \times 10^{-8}$ \\
\hline 0.15 & 0.52119617165177188 & 0.52119612312506341 & $4.85267 \times 10^{-8}$ \\
\hline 0.20 & 0.64880672544599941 & 0.64880668138466992 & $4.40613 \times 10^{-8}$ \\
\hline 0.25 & 0.76044150439367639 & 0.76044146588266251 & $3.85110 \times 10^{-8}$ \\
\hline 0.30 & 0.85335168974252162 & 0.85335165773009199 & $3.20124 \times 10^{-8}$ \\
\hline 0.35 & 0.92524952437801941 & 0.92524949965242641 & $2.47256 \times 10^{-8}$ \\
\hline 0.40 & 0.97436464499621131 & 0.97436462816628110 & $1.68299 \times 10^{-8}$ \\
\hline 0.45 & 0.99948767432373750 & 0.99948766580387880 & $8.51986 \times 10^{-9}$ \\
\hline 0.50 & 1 & 1 & 0 \\
\hline 0.55 & 0.97588900686653790 & 0.97588901538639662 & $8.51986 \times 10^{-9}$ \\
\hline 0.60 & 0.92774838759409579 & 0.92774840442402613 & $1.68299 \times 10^{-8}$ \\
\hline 0.65 & 0.85676352399871628 & 0.85676354872430928 & $2.47256 \times 10^{-8}$ \\
\hline 0.70 & 0.76468229900737327 & 0.76468233101980291 & $3.20124 \times 10^{-8}$ \\
\hline 0.75 & 0.65377205797941861 & 0.65377209649043262 & $3.85110 \times 10^{-8}$ \\
\hline 0.80 & 0.52676377913894699 & 0.52676382320027641 & $4.40613 \times 10^{-8}$ \\
\hline 0.85 & 0.38678482782732176 & 0.38678487635403025 & $4.85267 \times 10^{-8}$ \\
\hline 0.90 & 0.23728195038933994 & 0.23728200218653905 & $5.17972 \times 10^{-8}$ \\
\hline 0.95 & 0.08193640383912822 & 0.08193645763139898 & $5.37923 \times 10^{-8}$ \\
\hline 1.00 & -0.07542668890493687 & -0.07542663444213867 & $5.44628 \times 10^{-8}$ \\
\hline
\end{tabular}

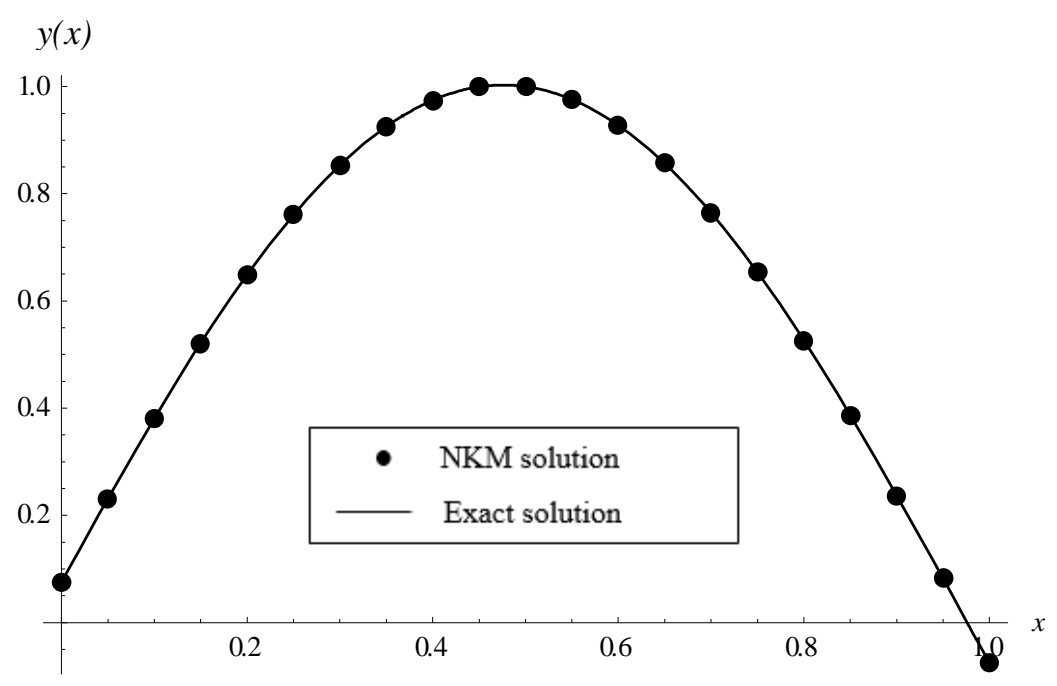

Fig. 1. Solutions to the integral equation (5)

(b) Adomian decomposition method

The exact solution of the equation (5) is $y(x)=$ $\sin (\pi x)+\frac{1}{3}(20-\sqrt{391}) \cos (\pi x)$.

In the following, we will calculate Adomian polynomials for the nonlinear terms $y^{3}(t)$ that arises in nonlinear integral equation.

By using the MATHEMATICA v9 software, and applying the procedure as stated above in equation (4f), we stop the iteration at the ninth step. Therefore we can write

$$
y(x)=\sin (\pi x)+\frac{9886326965781}{131072000000000} \cos (\pi x)
$$

The table below shows the approximate solutions obtained by applying the Adomian decomposition method according to the value of $x$, which is restricted between zero and one. We compared these results with the results which were obtained by the exact solution for this example, where the results are very close. Moreover, we can see the convergence of solutions in Figure 2, which is shown clearly. 
Table 2. Numerical and exact solutions of integral equation (5)

\begin{tabular}{|c|c|c|c|}
\hline Nodes & Exact values & Approximate values & Absolute Error \\
\hline 0.00 & 0.07542668890493687 & 0.07542668888687896 & $1.8057902 \times 10^{-11}$ \\
\hline 0.05 & 0.23093252624133365 & 0.23093252622349808 & $1.7835566 \times 10^{-11}$ \\
\hline 0.10 & 0.38075203836055493 & 0.38075203834338089 & $1.7174039 \times 10^{-11}$ \\
\hline 0.15 & 0.52119617165177188 & 0.52119617163568220 & $1.6089685 \times 10^{-11}$ \\
\hline 0.20 & 0.64880672544599941 & 0.64880672543139021 & $1.4609202 \times 10^{-11}$ \\
\hline 0.25 & 0.76044150439367639 & 0.76044150438090758 & $1.2768786 \times 10^{-11}$ \\
\hline 0.30 & 0.85335168974252162 & 0.85335168973190739 & $1.0614176 \times 10^{-11}$ \\
\hline 0.35 & 0.92524952437801941 & 0.92524952436982131 & $8.1981088 \times 10^{-12}$ \\
\hline 0.40 & 0.97436464499621131 & 0.97436464499063111 & $5.5802029 \times 10^{-12}$ \\
\hline 0.45 & 0.99948767432373750 & 0.99948767432091258 & $2.8249624 \times 10^{-12}$ \\
\hline 0.50 & 1 & 1 & 0 \\
\hline 0.55 & 0.97588900686653790 & 0.97588900686936292 & $2.8249624 \times 10^{-12}$ \\
\hline 0.60 & 0.92774838759409579 & 0.92774838759967579 & $5.5802029 \times 10^{-12}$ \\
\hline 0.65 & 0.85676352399871628 & 0.85676352400691436 & $8.1981088 \times 10^{-12}$ \\
\hline 0.70 & 0.76468229900737327 & 0.76468229901798749 & $1.0614176 \times 10^{-11}$ \\
\hline 0.75 & 0.65377205797941861 & 0.65377205799218751 & $1.2768786 \times 10^{-11}$ \\
\hline 0.80 & 0.52676377913894699 & 0.52676377915355617 & $1.4609202 \times 10^{-11}$ \\
\hline 0.85 & 0.38678482782732176 & 0.38678482784341145 & $1.6089685 \times 10^{-11}$ \\
\hline 0.90 & 0.23728195038933994 & 0.23728195040651389 & $1.7174067 \times 10^{-11}$ \\
\hline 0.95 & 0.08193640383912822 & 0.08193640385696378 & $1.7835566 \times 10^{-11}$ \\
\hline 1.00 & -0.07542668890493687 & -0.07542668888687896 & $1.8057902 \times 10^{-11}$ \\
\hline
\end{tabular}

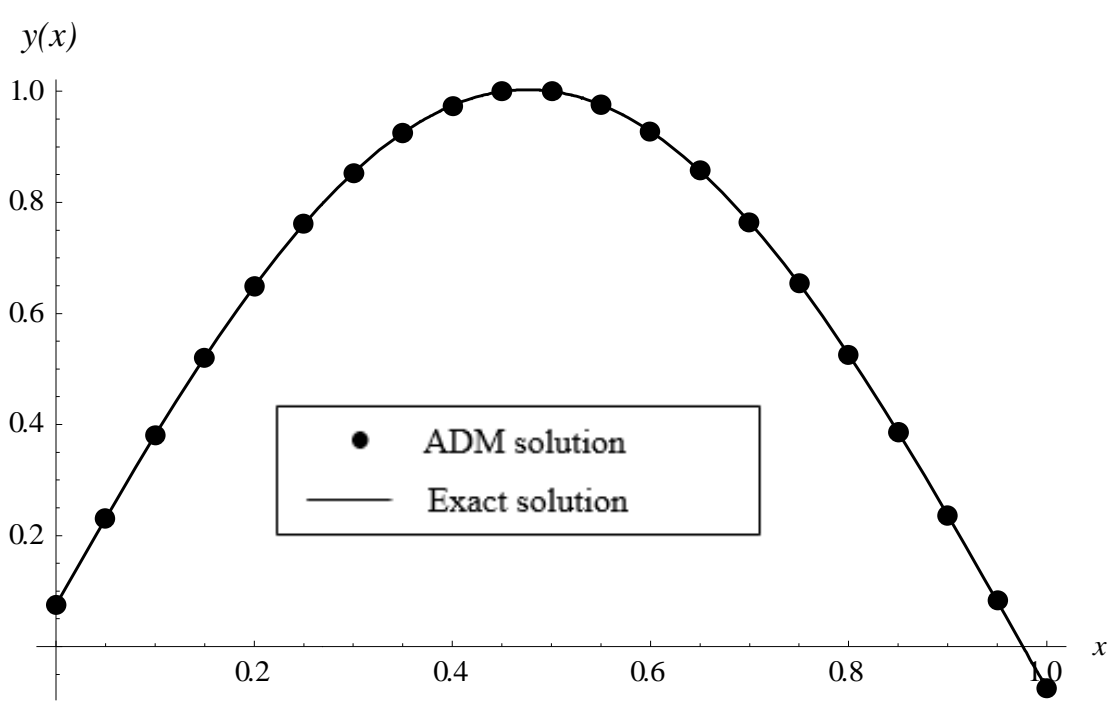

Fig. 2. Solutions to the integral equation (5)

Comparison results between Newton-Kantorovich quadrature method and Adomian decomposition method

The numerical results show the following conclusion:

In example, we have applied the following methods: Newton-Kantorovich and Adomian decomposition. We have obtained the following results:

\begin{tabular}{|c|c|}
\hline $\begin{array}{c}\text { Numerical method } \\
\text { The Newton-Kantorovich } \\
\text { method }\end{array}$ & Maximum absolute error \\
\hline $\begin{array}{c}\text { The Adomian } \\
\text { decomposition method }\end{array}$ & $1.8057902 \times 10^{-11}$ \\
\hline
\end{tabular}


From the above table we see clearly that Adomian decomposition method is the most efficient method for solving the integral equation (5).

\section{Conclusion}

Integral equations occur naturally in many fields of science and engineering. In this paper, we have applied the NKM and ADM to solve nonlinear Fredholm integral equation. The accuracy of the numerical results indicates that the Adomian decomposition method is well suited for the solution of such type of problems. The advantages of current approach are that it provides a direct scheme for obtaining approximations of the solutions. The numerical results show that only a few terms are required to obtain accurate solutions.

\section{References}

1. Adomian G., 1994. Solving Frontier Problem: The Decomposition Method, Kluwer: Dordrecht.

2. Adomian G., \& R. Rach, 1992. Nonlinear transformation of series - Part II, Computational Mathematics Applications. 23, 79-83.

3. Ghorbani A., 2009. Beyond Adomian polynomials: He polynomials, Chaos, Solutions \& Fractals, 39, (3), 1486-1492.
4. Ghorbani A. and J. Saberi-Nadjafi, 2007. He's homotopy perturbation method for calculating adomian polynomials, International Journal of Nonlinear Sciences and Numerical Simulation, 8, (2), 229-232.

5. Jerri A. A., 1985. Introduction to Integral Equations with Applications, Marcel Dekker, Inc, New York, 15, 155, 188200.

6. Mahmoud Paripour and Mahdi Kamyar, 2013. Numerical Solution of nonlinear Volterra-Fredholm integral equations by using new basis functions, International Scientific Publications and Consulting Services.

7. Nadjafi J. S. and M. Heidari, 2010. Solving Nonlinear IntegralEquations in the Urysohn form by NewtonKantorovich-Quadrature Method, Computers and Mathematics with Applications, (60), 2058-2059.

8. Porshokouhi M. G. and B. Ghanbari and B. Rahimi, 2011. Numerical Solution for Non-Linear Fredholm Integral Equations by Newton- Kantorovich Method and Comparison with HPM and ADM, International Journal of Pure and Applied Sciences and Technology, (3), 44-49.

9. Wazwaz, A. M. \& R. Rach, 2011. Comparison of the Adomian decomposition method and the variational iteration method for solving the Lane-Emden equations of the first and second kinds, Kybernetes, 40, Issue(s) 9/10, 1305-1318. 